\title{
Dynamic Brachytherapy of the Prostate Under Active Image Guidance
}

\author{
Gang Cheng ${ }^{1}$, Haisong Liu ${ }^{1}$, Lydia Liao ${ }^{2}$ and Yan Yu ${ }^{1}$ \\ Departments of ${ }^{1}$ Radiation Oncology and ${ }^{2}$ Radiology, University of Rochester, \\ 601 Elmwood Avenue, Rochester, NY 14642, USA
}

\begin{abstract}
Image-guided brachytherapy is a promising treatment for early stage prostate cancer. Current research emphasizes methods for intraoperative optimized planning and precise implantation of radioactive seeds. Some new technologies for these purposes are described in this paper. A morphological template will overcome pubic arch interference and achieve better-optimized dose coverage under the real-time dynamic dosimetry technique. Autosegmentation of the prostate anatomy has been implemented for clinical use to reduce operative time. Just-in-time planning and procedure tracking under active image guidance can now be performed in the operating room. Interactive planning and dynamic dosimetry can be achieved based on seed recognition in the live ultrasound images, which permit real-time replanning to eliminate under-dosage. As a result of these advances, prostate brachytherapy of the future will increasingly be practised as a precision procedure rather than an art form.
\end{abstract}

\section{Introduction}

In the United States, adenocarcinoma of the prostate is the most common malignancy in man, excluding skin cancer. Prostate cancer is newly diagnosed in over 180,000 men in the US each year. Growing emphasis on prostatic specific antigen (PSA) based early detection and changes in the population demographics suggest that prostate cancer diagnosis, particularly of the early stages, will continue to increase. Standard treatment options at this time for early, localized prostate cancer include prostatectomy, external beam radiation with or without $3 \mathrm{D}$ conformal intensity modulation, and interstitial brachytherapy using either permanent implantation of radioactive seeds or temporary placement of high dose rate needle channels. Whereas surgery and external beam radiation therapy offers effective treatment for localized disease, both treatment modalities can cause significant side effects such as urinary incontinence and impotence. In the last few years, there is a rapid growth of interest in interstitial brachytherapy with ${ }^{125} \mathrm{I}$ and ${ }^{103} \mathrm{Pd}$ seeds under the guidance of live ultrasound and needle template. However, it is now widely recognized that interstitial brachytherapy of the prostate is susceptible to great variability due to factors in patient anatomy, dosimetry optimization, and precision of needle placement. Current research emphasis in prostate brachytherapy is therefore to develop methods for intraoperative optimized planning and precise implantation of radioactive seeds for real-time, image-guided prostate brachytherapy. 
In modern prostate brachytherapy, computed tomography (CT), magnetic resonance imaging (MRI) and transrectal ultrasound (TRUS) image guidance have been used to replace free-hand seed implantation. In particular, TRUS permits real time localization of the prostate gland and the needle locations at the same time, which leads to stepwise updating of the actual radiation dosimetry that reflects needle departures from the idealized plan.

\section{Treatment Planning Space}

The current implantation guidance template is a rectilinear uniform design permitting parallel needle tracks at uniform ( $5 \mathrm{~mm}$ interval) spacing in the left-right and anteriorposterior directions. The square template pattern is an anatomically arbitrary design reflective of the static grid that the early generations of ultrasound machines were capable of displaying on screen. This design artifact led to a long practice of so-called "uniform loading," which for prostate brachytherapy caused excessive dosage to the urethra and the central portions of the prostate gland. Pubic arch interference (PAI) occurs in patients with large prostates where the anterior and/or lateral aspects of the prostate gland are blocked by the pubic bone in the transperineal approach. Although improvisation techniques have been applied to tilt the pelvis in an ad hoc manner, the vast majority of treatment protocols would exclude patients with prostate size greater than 55 to $60 \mathrm{cc}$, who would undergo hormonal downsizing to reduce the prostate size and therefore the degree of PAI. Though Roy and Wallner developed a methodology permitted semi-automatic optimization of dosimetry and pubic arch avoidance by allowing needle tracks to be positioned with considerable flexibility including angulation, their individual customization of the template does not offer a class solution to the prostate morphology for universal applicability. ${ }^{[1]}$

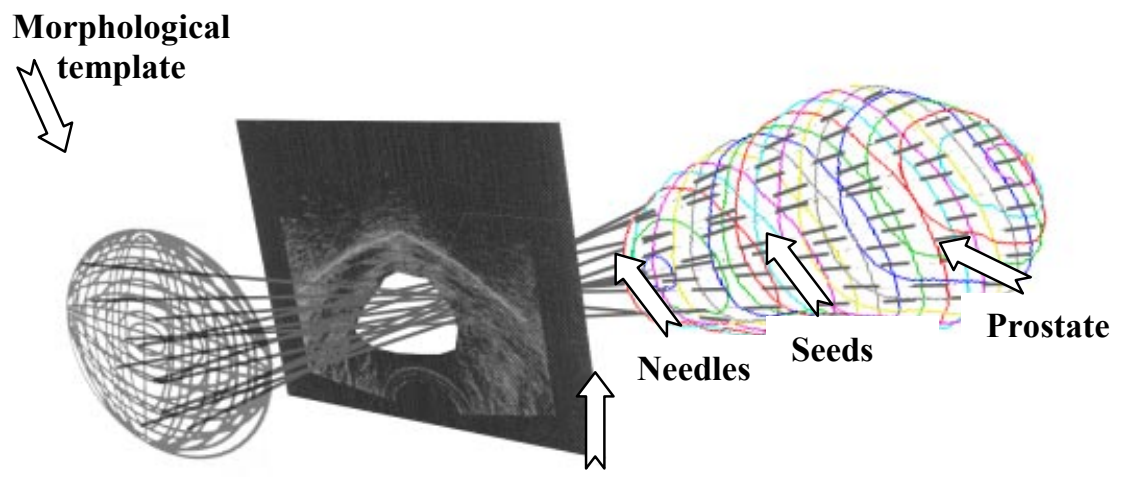

PAI in TRUS image

Fig. 1. The morphological template will open up a large solid angle of the implant space for the most effective replanning and optimization in real time. 
A morphological template concept (Fig. 1) is being developed as part of the PIPER (Prostate Implant Planning Engine for Radiotherapy) project, ${ }^{[2]}$ which leverages the planning system's active sign guidance capabilities to deliver noncoplanar conformal interstitial brachytherapy. The morphological template will allow patients with pubic arch interference to be treated with brachytherapy or other interstitial ablative therapies of the prostate without first undergoing lengthy hormonal downsizing therapy. In addition, the non-coplanar conformal needle pattern will open up greater degrees of freedom for optimized planning as well as error-feedback/feedforward dynamic brachytherapy. Dynamic re-optimization of the dosimetry plan during brachytherapy seed placement based on real time automatic localization of the prostate boundaries and the implanted seeds on TRUS would benefit from needle adjustments around the periphery of the target volume on the morphologically spaced template where under-dosage usually occurs.

\section{Auto-segmentation of Prostate Gland}

Prostate volume study using TRUS is an important step for planning optimized dosimetry. In the volume study, transverse cross-sectional images of the prostate are acquired at fixed intervals, e.g., $5 \mathrm{~mm}$ increments from the base (superior) of the gland to the apex (inferior). The boundaries of the prostate obtained during the volume study not only result in an accurate determination of the size and shape of the prostate, but also provide important information for adequate dose delivery to the target volume with sparing of dose-sensitive tissues.

Currently, the prostate boundary is manually outlined in each transverse crosssectional ultrasound image, which is a tedious and time-consuming process, particularly for intraoperative planning. Automatic segmentation is a difficult task due to the inherent noise in the ultrasound image data. However, recent advances in software methodology have made remarkable progress in this area. Arambula has used a genetic algorithm and a constrained prostate model to automatically identify the prostate in ultrasound images. ${ }^{[3]}$ Kwoh used the harmonics method and Radial Bas-Relief (RBR) method to extract a skeletonised boundary from an ultrasound image automatically. ${ }^{[4]}$ Aarnink has investigated multi-scale edge detection algorithm and nonlinear Laplace filtering to improve edge localization in ultrasound images of the prostate. ${ }^{[5][6]}$ We have developed a trainable method for TRUS segmentation and demonstrated its utility in prostate brachytherapy planning and optimization. ${ }^{[7]}$ In this method, the initial ultrasound images are pre-processed to remove noise and increase the contrast. The rectum edge is located from the bottom of the images. Key points on the prostate boundaries are located and connected under the iterative training of a knowledge-based model until the shape of the boundary reaches a stable state. The urethra is segmented near the center of the prostate. In our initial assessment, the automated image segmentation technique will reduce the median operative time for anatomy delineation for intraoperative planning, from $10 \mathrm{~min}$. to $2 \mathrm{~min}$. for complete outline of the prostate, urethra and rectum (Fig. 2). 


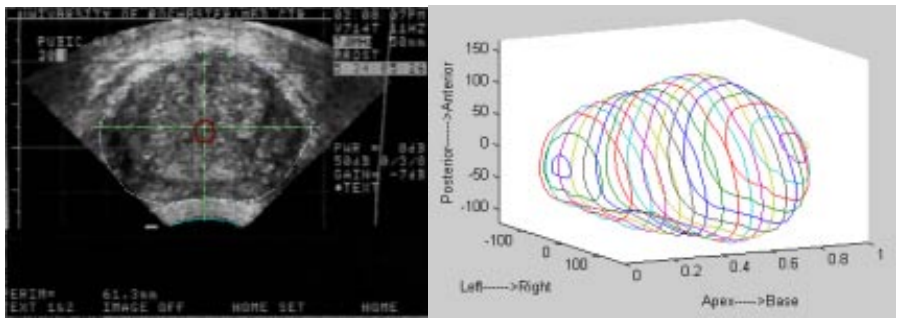

(a) 2D auto-segmentation of prostate gland. (b) 3D reconstruction of prostate surface.

Fig. 2. Auto-segmentation of the prostate gland and 3D reconstruction.

\section{Intraoperative Planning Engine}

It is now widely recognized that the two-step process of pre-planning (approximately 2-4 weeks prior to implantation) and surgical implantation had several limitations: (a) the pre-planning prostate position often cannot be precisely reproduced in the operating room, especially following relaxation of the pelvic musculature due to anesthesia; (b) time lapse and/or interim hormonal therapy can significantly change the prostate size and shape. These factors limit the efficacy of the pre-plan, often rendering it invalid. Strict adherence to the pre-plan may lead to severe under-dosage and local failure, whereas ad hoc improvisation at the time of implantation can lead to unexpected morbidity.

Compared to pre-planning, the intraoperative planning system refers to the creation of a plan in the operation room just before the implant procedure with immediate execution of the plan, which eliminates separate planning image study with resultant improved efficiency and less patient inconvenience, and reduces the dependence on pubic arch obstruction evaluation study. At the same time, intraoperative planning eliminates the need to reproduce patient and ultrasound probe position as ultrasound images are captured in the OR immediately prior to and during the implant with patient in the treatment position so that it can account for changes in size and shape of the prostate due to hormonal therapy or muscle relaxation. Consequently the best match is obtainable between the planned seed configuration and the actual seed placement locations in the prostate. Thus intraoperative planning reduces the chain of uncertainties to a minimum, thereby minimizing the chance of under-dosage to parts of the cancerous prostate. Real-time dosimetric guidance is a natural extension of intraoperative optimized planning. Based on the use of computer and optimization technologies, dosimetric guidance requires an additional feedback loop following real-time acquisition of seed placement/prostate volume data at selected intervals (e.g. after every 4 needle insertions).

Systematic investigation of treatment planning, seed placement uncertainties and dosimetric consequences of realistic (rather than idealized) implants in the new era of TRUS- and template-guided prostate brachytherapy began in $1993,{ }^{[8]}$ followed by 
rigorous efforts to approach dosimetric planning using computerized optimization techniques. ${ }^{[9]}$ This work was later extended to account for the multi-objective nature of treatment planning, including delivering therapeutic dosage to the entire prostate and higher intensity dosage to the tumor foci, sparing the urethra, rectum and neurovascular bundles, and reducing the complexity of needle/seed placement plans. ${ }^{[10]}$ GA optimization and multi-objective decision theory embedded in these early works form the basis of the PIPER (Prostate Implant Planning Engine for Radiotherapy) system for both web-based Internet treatment planning and intraoperative real time dosimetry planning. ${ }^{[2][11]}$ Differential planning to the PTV (planning target volume), GTV (gross tumor volume) and GTF (gross tumor foci) is achievable under the multi-objective optimization framework used by PIPER. In the pilot clinical experience, we demonstrated that intraoperative just-in-time planning using PIPER reduced dosimetric variance that otherwise would occur due to such variabilities as patient pelvic positioning, interim prostate volume change between preplanning and the procedure, and acute edema following the placement of the first three stabilizing needles. ${ }^{[2]}$ Figure 3 shows screen shots of the PIPER system demonstrating the main functionalities designed according to the workflow in actual brachytherapy procedures.

The current PIPER system is designed to connect with the ultrasound machine next to the operating table. When the patient is positioned after anaesthesia induction, ultrasound images of the prostate from base to apex are acquired in registration with the implant template coordinates. The pelvic anatomy is then defined on the images, as shown in Fig. 3a. The anatomy data and the basic radiation dosimetry data are used in the GA planning engine to produce a dose distribution that best complies with the treatment intent, such as prescription dose, dosimetric margin requirements and relative importance of sparing each of the critical structures (Fig. 3b). The needle and seed patterns generated by this treatment planning process are followed during the procedure in an innovative needle tracking design unique to PIPER (Fig. 3c): the live TRUS video signal is imported to the planning system in a real time video window (top right). As each needle insertion occurs, a hyperechoic spot can be identified in this window and is immediately localized. Because the live ultrasound is registered with the planning coordinate system and the template and the planned needle coordinates are accurately known, the needle positioning error in $\mathrm{x} / \mathrm{y}$ displacements and angular splaying can be quantified immediately. The physician has the option of either accepting the deviations or reinserting the needle. The isodose coverage of the prostate is automatically updated to account for the needle misplacement. It is now a well-known quality problem in prostate brachytherapy that no implant can achieve the planned dose coverage, and that the magnitude of under-dosage to the prostate and/or over-dosage to radio-sensitive critical structures are unknown at the end of the procedure. Implant dosimetry analysis is currently performed using CT post-implant (Fig. 3d); by that time it is already impractical to re-implant sub-optimal treatment or to extract seeds that will cause complications. The needle-tracking feature is the first practical technique to enable online intraoperative dose evaluation and decisionmaking in ultrasound-guided brachytherapy. 


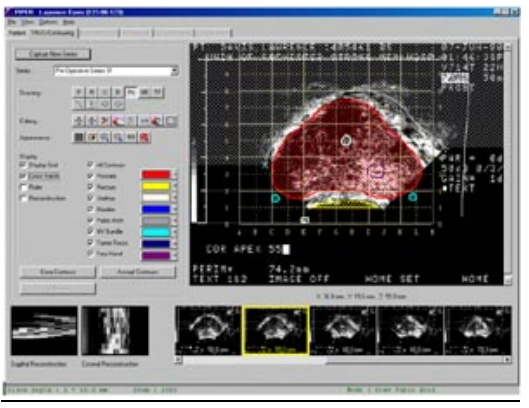

(a)

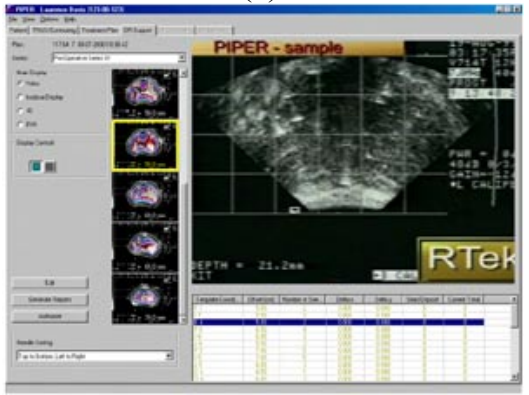

(c)

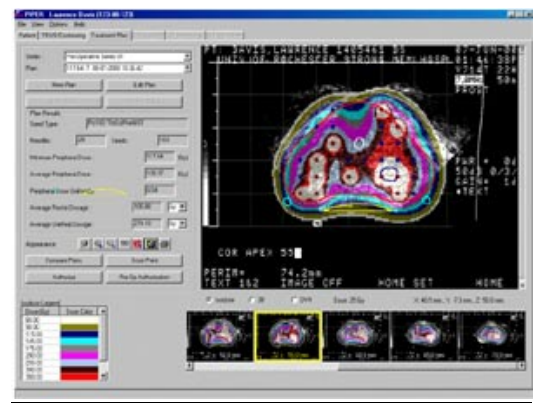

(b)

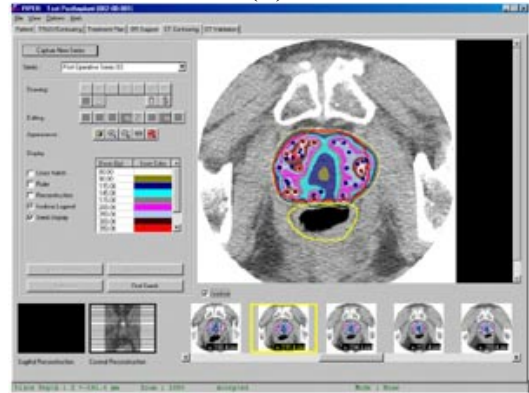

(d)

Fig. 3. PIPER in intraoperative use.

\section{Dose Delivery and Dynamic Brachytherapy}

\subsection{Interactive Planning and Dynamic Dosimetry}

Since 1997, a new paradigm of error-feedback/feedforward, dynamic brachytherapy under active, "smart" image guidance was introduced at the University of Rochester. This system continuously acquires volume ultrasound data of the prostate throughout the procedure, perform automatic recognition of the prostate and critical structures, automatically locate each seed as it is implanted into the prostate and perform dosimetry analysis on the fly. During implant progression, an error distribution is computed by comparing the intended seed positions and the detected positions. If the original treatment plan has suffered too much deviation that isolated additional seed placement is not expected to lead to adequate therapy, the GA planning engine can be rerun in the available needle space but taking into account the dosimetric contribution from seeds already implanted in the patient. Dynamic optimization of subsequent seed configurations will take into account the likelihood and extent of misplacement by the convolution of sensitivity analysis and genetic algorithm. Thus the ultimate goal is not only to deliver on-the-fly dosimetric analysis and visualization, but also to recommend subsequent seed placement that anticipates surgical uncertainties rather than chasing after cold spots. 


\subsection{Active Guidance of Implantation Needles}

Targeting of non-coplanar needle placement requires visual guidance on live TRUS, so active guidance sign projected on screen based on the known ultrasound probe locations in space is useful to facilitate pinpoint accuracy in targeted needle placement in any ultrasound view plane at any time.

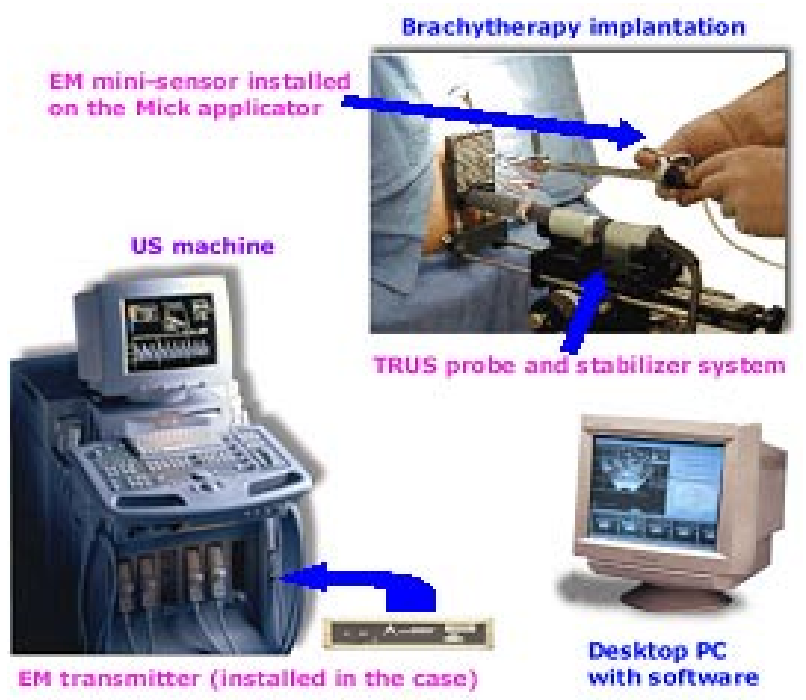

Fig. 4. EM tracking system in the OR.

In PIPER, the electromagnetic (EM) positioning system (Fig. 4) will obtain the $3 \mathrm{D}$ physical positions of the needle and the TRUS probe at the same time so that precise registration between the needle and the prostate will be obtained in the physical coordinate system as established by the tracking system. The positions of the needle will be recognized and displayed on the screen with the TRUS image. An active image-subtraction algorithm is developed to analyze whether the needle reaches the pre-planned positions; corresponding instructions will be displayed on the screen at the same time to assist the physician-user in inserting the needle. The active sign and instruction will be shown on the screen to inform the physician-user of the correct steps. Once a needle shadow is found, the TRUS probe will move to the next position unless this needle insertion is completed. If a needle shadow cannot be detected in the viewing window, the needle should be adjusted according to the instructions displayed on the screen based on active seek of the needle path by the tracked ultrasound probe. (Fig. 5) 


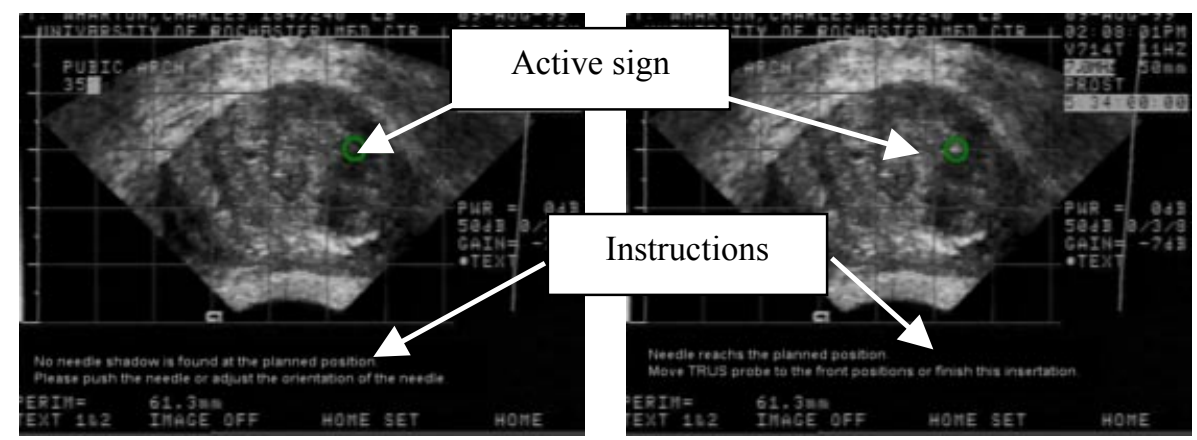

Fig. 5. The active guidance of the needle insertion, (a) The active sign and instruction before the needle insertion; (b) The active sign and instruction after the needle insertion.

\subsection{Intraoperative Seed Recognition}

Tracking of needle pathways is a first-order dosimetry correction in realistic brachytherapy. It provides the basis for higher-order, seed-based dosimetry necessary in dynamic brachytherapy. After the seeds are implanted in tissue, each seed located in the column of images is recognized by an adaptive-tracking recognition method and given a confidence level, which shows the accuracy of the seed recognition in view of the large noise interference in the transrectal ultrasound images. The dosimetry is recalculated based on the recognized seeds. Periodically throughout the seed placement and at the end of seed placement, fluoroscopic x-rays are taken, and the seed coordinates found in transrectal ultrasound images are matched to the seed coordinates found in fluoroscopic x-ray image. Seed locations with low confidence levels are adjusted based on the x-ray locations, and the dosimetry is recalculated based on these revised seed positions (Fig. 6).

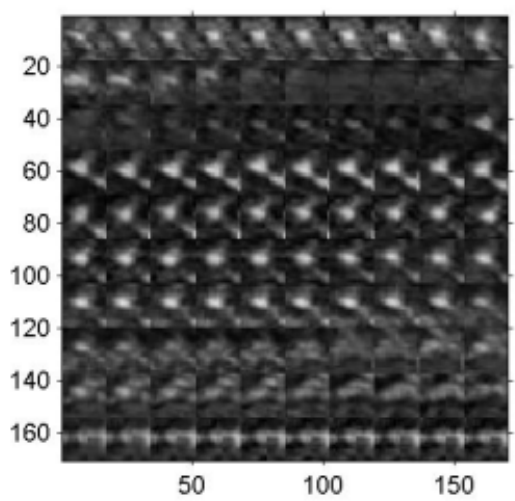

I2 Transverse View (Looking from Apex to Base)

Fig. 6. Seed pathway Tracking (left: original sub-images; right: highlighted seed tracks). 
Because angulated needle tracks, used in morphological template, do not necessarily follow any major anatomical planes (e.g., sagittal, coronal), active seek and active guidance of the needle insertion are implemented on live $2 \mathrm{D} / 3 \mathrm{D}$ ultrasound, using the needle path recognition algorithm and standard triangulation methodology.

\section{Summary and Conclusion}

Active image-guidance technologies are now in place to achieve dynamic and quantitative brachytherapy of the prostate in operative conditions with greatly increased surgical implant space and optimized real-time dosimetry planning.

\section{References}

1. Roy, J. N., Wallner, K. E., Chiu-Tsao, S. T., Anderson, L. L., and Ling, C. C.: CT-based optimized planning for transperineal prostate planning with customized template. Int. J. Radiat. Oncol. Biol. Phys. 21 (1991) 483-489.

2. Messing, E.M., Zhang, J.B., Rubens, D.J., Brasacchio, R.A., Strang, J.G., Soni, A., Okunieff, P.G. and Yu, Y.: Intraoperative optimized inverse planning for prostate brachytherapy: Early experience. Int. J. Radiat. Oncol. Biol. Phys. 44 (1999) 801-808.

3. Arambula, C. F., Davies, B.L.: Automated prostate recognition: a key process for clinically effective robotic prostatectomy. Med Biol Eng Comput Mar. 37 (1999) 236243.

4. Kwoh, C. K., Teo, M. Y., Ng, W. S., Tan, S. N., Jones, L. M.: Outlining the prostate boundary using the harmonics method. Med Biol Eng Comput. 36 (1998) 768-771.

5. Aarnink, R. G., Pathak, S. D., de la Rosette, J. J., Debruyne, F. M., Kim, Y., Wijkstra, H.: Edge detection in prostatic ultrasound images using integrated edge maps. Ultrasonics. 36 (1998) 635-642.

6. Aarnink, R. G., Giesen, R. J., Huynen, A. L., de la Rosette, J. J., Debruyne, F. M., Wijkstra, H.: A practical clinical method for contour determination in ultrasonographic prostate images. Ultrasound Med Biol. 20 (1994) 705-717.

7. Cheng, G., Liu, H., Rubens, D.J., Strang, J.G., Liao, L., Yu, Y., Brasacchio, R., Messing, E.: Automatic segmentation of prostate in transrectal ultrasound imaging. Radiology 218 (2001) 612.

8. Yu, Y., Waterman, F.M., Suntharalingam, N., Schulsinger, A.: Limitations of the minimum peripheral dose as a parameter for dose specification in permanent ${ }^{125}$ I prostate implants. Int. J. Radiation Oncology Biol. Phys. 34 (1996) 717-725.

9. Yu, Y. and Schell, M.C.: A genetic algorithm for the optimization of prostate implants. Med. Phys. 23 (1996) 2085-2091.

10. Yu, Y.: Multiobjective decision theory for computational optimization in radiation therapy. Med. Phys. 24 (1997) 1445-1454.

11. Yu, Y., Zhang, J. B., Brasacchio, R.A., Okunieff, P.G., Rubens, D.J., Strang, J.G., Soni, A., and Messing, E.M.: Automated treatment planning engine for prostate seed implant brachytherapy. Int. J. Radiat. Oncol. Biol. Phys. 43 (1999) 647-652. 